\title{
Cost-effectiveness of a community-based cardiovascular disease prevention intervention in medically underserved rural areas
}

Hua Wang ${ }^{1 *}$, Donald Kenkel ${ }^{2}$, Meredith L. Graham ${ }^{3}$, Lynn C. Paul ${ }^{4}$, Sara C. Folta ${ }^{5}$, Miriam E. Nelson, David Strogatz ${ }^{7}$ and Rebecca A. Seguin ${ }^{8}$

\begin{abstract}
Background: Rural women experience health disparities in terms of cardiovascular disease (CVD) risk compared to urban women. Cost-effective CVD-prevention programs are needed for this population. The objective of this study was to conduct cost analysis and cost-effectiveness analyses (CEAs) of the Strong Hearts, Healthy Communities (SHHC) program compared to a control program in terms of change in CVD risk factors, including body weight and quality-adjusted life years (QALYs).

Methods: Sixteen medically underserved rural towns in Montana and New York were randomly assigned to SHHC, a six-month twice-weekly experiential learning lifestyle program focused predominantly on diet and physical activity behaviors $(n=101)$, or a monthly healthy lifestyle education-only control program $(n=93)$. Females who were sedentary, overweight or obese, and aged 40 years or older were enrolled. The cost analysis calculated the total and per participant resource costs as well as participants' costs for the SHHC and control programs. In the intermediate health outcomes CEAs, the incremental costs were compared to the incremental changes in the outcomes. The QALY CEA compares the incremental costs and effectiveness of a national SHHC intervention for a hypothetical cohort of 2.2 million women compared to the status quo alternative.
\end{abstract}

Results: The resource cost of SHHC was $\$ 775$ per participant. The incremental cost-effectiveness ratios from the payer's perspective was $\$ 360$ per $\mathrm{kg}$ of weight loss. Over a 10-year time horizon, to avert per QALY lost SHHC is estimated to cost $\$ 238,271$ from the societal perspective, but only $\$ 62,646$ from the healthcare sector perspective. Probabilistic sensitivity analyses show considerable uncertainty in the estimated incremental cost-effectiveness ratios.

Conclusions: A national SHHC intervention is likely to be cost-effective at willingness-to-pay thresholds based on guidelines for federal regulatory impact analysis, but may not be at commonly used lower threshold values. However, it is possible that program costs in rural areas are higher than previously studied programs in more urban areas, due to a lack of staff and physical activity resources as well as availability for partnerships with existing organizations.

Trial registration: ClinicalTrials.gov identifier NCT02499731, registered on July 16, 2015.

Keywords: Cost-effectiveness analysis, Cardiovascular disease prevention, Rural, Economic evaluation, Quality adjusted life years, Women

\footnotetext{
* Correspondence: hw227@cornell.edu

'Cornell University, 292 Martha Van Rensselaer Hall, Ithaca, NY 14853, USA

Full list of author information is available at the end of the article
}

(c) The Author(s). 2019 Open Access This article is distributed under the terms of the Creative Commons Attribution 4.0 International License (http://creativecommons.org/licenses/by/4.0/), which permits unrestricted use, distribution, and reproduction in any medium, provided you give appropriate credit to the original author(s) and the source, provide a link to the Creative Commons license, and indicate if changes were made. The Creative Commons Public Domain Dedication waiver (http://creativecommons.org/publicdomain/zero/1.0/) applies to the data made available in this article, unless otherwise stated. 


\section{Background}

Cardiovascular disease (CVD) accounts for about one-third of all deaths in the U.S. and is the leading cause of mortality [1]. Rural populations face CVD-related health disparities compared to their urban counterparts; they are less likely to meet physical activity recommendations and more likely to smoke, be overweight, and have type 2 diabetes [2, 3]. Rural women face additional health risk factors in terms of income, education, age, and insurance [2]. Environmental aspects of rural areas, including limited access to physical activity opportunities, healthy foods, and healthcare resources, contribute to development of these risk factors [4-6]. Therefore, women in rural, medically underserved areas are a critical population for CVD prevention interventions.

Health policy makers face difficult choices between funding targeted CVD prevention efforts, other public health efforts, and clinical healthcare. Economic evaluation methods are used to guide scarce societal resources to their highly valued use in improving health $[7,8]$. Investments in prevention can be particularly attractive when they reduce the need for future clinical healthcare spending [9]. More often, prevention adds to healthcare costs, but can still provide an attractive return on investment compared to other options to improve health [10].

Community-based CVD, type 2 diabetes, and obesity prevention programs are generally found to be cost-effective [11-13] Because midlife and older rural women face health disparities and rural areas may lack healthy lifestyle resources, CVD prevention programs are critical for this population. Relatively few cost-effectiveness analyses (CEAs) have been performed for community-based healthy lifestyle interventions in rural areas [14-18]. Some of these previous studies were not randomized controlled trials [14, 18], combined results from urban and rural areas [14], had under 50 participants $[17,18]$ or did not report the number of participants [14], included men and women [14, 15, 18], and/or included young adults, as well as midlife and older adults $[15,17]$. Only one previous CEA has been done on a community-based healthy lifestyle behavior change program studying only midlife and older rural women, in the United States, with over 50 participants [16].

In this paper, we report the results of economic evaluations of a rural community-based CVD prevention program targeted at overweight and obese rural women aged 40 and older. Strong Hearts, Healthy Communities (SHHC) was an innovative community-based six-month intervention informed by the socioecological framework to target key behaviors related to CVD prevention and overweight/obesity. We hypothesized that the cost-effectiveness of SHHC would compare favorably with other interventions and commonly used thresholds for acceptable costs per quality-adjusted life year (QALY) saved.

\section{Methods \\ Research design}

We conducted economic evaluations of SHHC alongside a two-armed randomized controlled efficacy trial. The economic evaluations include a program cost analysis (CA) and CEAs that adopted alternative perspectives and used multiple health outcome measures. The study protocol for the efficacy trial has been previously published [19]. The study was approved by the Cornell University and Bassett Healthcare Institutional Review Boards.

The efficacy trial compared the multilevel SHHC intervention to a minimal, education-only control program, Strong Hearts, Healthy Women (CON). Randomization occurred at the town level, with half randomized to deliver the SHHC intervention program and half to deliver the CON program. The primary outcomes of the efficacy trial were $\mathrm{kg}$ of body weight and BMI; physiological measures and two composite measures of cardiovascular disease risk were also evaluated [20].

\section{Participants and setting}

Sedentary overweight or obese women aged 40 and older were recruited from 16 medically underserved rural towns in Montana and New York. Participants were recruited by local health educators. Eligible participants were female, 40 years or older, overweight (BMI > 25), sedentary, English-speaking, and had their physician's approval to participate. Participants with blood pressure $>160$ (systolic) or $>100$ (diastolic), heart rates of < 60 or $>100$, or cognitive impairments were excluded. All participants provided written informed consent. A total of 436 participants were assessed for eligibility; 194 participants enrolled.

\section{Interventions}

Based on previous effective programs [21-23], the SHHC multilevel CVD prevention program targeted individual (experiential learning around dietary intake and physical activity, including aerobic exercise and strength training), social (activities including family and friends), and community (civic engagement curriculum designed to catalyze positive built environment) levels. SHHC classes met for 1 hour twice weekly for 24 weeks (48 classes total).

The Strong Hearts, Healthy Women control (CON) classes served as the reduced-dose, education-only, minimal intervention control program. Classes provided evidence-based healthy lifestyle information (e.g. current dietary and physical activity guidelines) presented didactically. Participants did not engage in physical activity, 
skill building, or other active learning elements (e.g. reflection, monitoring) or civic engagement during the class sessions. The CON classes met for a one-hour class once per month over 24 weeks (six classes total).

\section{Perspectives of the CEA}

The CA and one set of CEAs were conducted from the payer perspective, which means we estimated how much the intervention's payer or sponsor paid for the intervention. When we adopted the payer perspective, we focused on costs directly incurred to administer and implement the program. The results of the payer perspective analysis provide crucial information for local health policy makers to judge whether and under what circumstances SHHC should be disseminated.

As recommended by the Second Panel on Cost-Effectiveness in Health and Medicine [7], we also conducted Reference Case CEAs from two broader perspectives. The Reference Case CEAs from the broad societal perspective consider all significant health outcomes and costs, including participants' direct and opportunity costs, that flow from the intervention. The Reference Case CEAs from the healthcare sector perspective consider formal healthcare sector (medical) costs borne by third-party payors or paid out-of-pocket by patients. The results of the Reference Case analyses allows comparison of the cost-effectiveness of SHHC to existing cost-effectiveness research on a broad range of prevention and clinical interventions.

\section{Health outcomes}

In one set of CEAs, we used the health outcomes measured in the efficacy trial. In these CEAs we examined the cost per $\mathrm{kg}$ of body weight reduction; the cost per point of $\mathrm{BMI}$ reduction; the cost per $\mathrm{mg} / \mathrm{L}$ of $\mathrm{C}$-reactive protein (CRP) reduction; and the cost per point of Simple 7 increase. Simple 7 is a composite cardiovascular health metric composed of four health behaviors (non-smoking, appropriate BMI, physical activity, healthy diet) and three health factors (total cholesterol, blood pressure, fasting glucose) [24].

In another CEA we used the ten-year risk for atherosclerotic cardiovascular disease (ASCVD) to conduct CEA in terms of cost per QALY saved. ASCVD risk was calculated using the Pooled Cohort Equations based on age, total cholesterol, high-density lipoprotein cholesterol, systolic blood pressure (including treated or untreated status), diabetes, and current smoking status [25]. We used previously published estimates to calculate the QALYs saved per ASCVD event prevented [26].

\section{Costs}

For the CA and the CEAs from the payer perspective, we identified the resources directly used in program administration and implementation and collected measures of the associated tangible costs. The categories of resource use were labor, facilities (space and utilities), food, equipment, curriculum printing, and other. Labor resources include staff training and preparation time, as well as staff time to deliver the interventions. Information on resource use was provided as administrative records or collected via surveys of the program administrators. Administrative records on staff compensation including salary and fringe benefits were used to calculate labor costs. Administrative records also provided the information on the costs of food, equipment, and curriculum printing. The labor and food costs are provided at the site-level. The equipment and printing costs are overall estimates. The space rental fees and other costs are estimated based on a dataset from a survey of agents and coordinators. Site level costs are taken from administrative records (labor, food, equipment, and printing) or estimated as site level means from surveys (rent, other costs, and participants' gasoline and time). Missing site level costs for labor, rent, and others are replaced by the mean cost per site within the SHHC or CON program. Missing food and participant costs are estimated based on the mean cost per enrollee per site within program. The total costs for SHHC and CON are the sum of all their sites' costs.

For the Reference Case CEAs from the societal perspective, we measured not only the direct program resource costs, but also the opportunity cost of all resources used as a result of the intervention. Costs to participants are an important component of the opportunity costs included from the societal perspective. Participants give up time that could have been used in other valued ways such as labor market work, household work, or leisure activities. We collected information on participant costs from surveys of participants. We followed standard practice and measured the value of participants' time based on the relevant wage rates.

For the Reference Case CEAs from the healthcare sector perspective, we used estimates of the medical costs of CVD events [27]. The estimates are from a study that used administrative claims data from a large U.S. health plan to predict medical costs of coronary heart disease and stroke events.

\section{Analyses}

The CA calculated the total and per participant costs of the resources used in the administration and implementation of the SHHC and CON interventions. The CEAs calculate incremental cost-effectiveness ratios (ICERs) as the ratio of the incremental costs over the incremental effectiveness.

In the intermediate health outcomes CEAs, the incremental costs were calculated as the per participant costs 
in the SHHC intervention minus the per participant costs in the CON. The incremental effectiveness estimates were taken from the between group multivariate analysis of the SHHC intervention's impact compared to the CON on weight, BMI, CRP, and Simple 7 score [20].

In the QALY CEA, the incremental costs and effectiveness compare the SHHC intervention to the status quo alternative (no intervention). We conducted the analysis for a hypothetical cohort of 2.2 million women. The cohort size corresponds to a hypothetical nationwide SHHC intervention that reaches all midlife and older overweight and obese women in rural medically underserved communities [28]. We used the incidence rates for 55-64 year-old women to predict the status quo number of CVD events in a cohort this size over a 10 -year time horizon. We predicted the number of CVD events after the hypothetical SHHC intervention based on the pre-post within group multivariate analysis of the SHHC's intervention impact on ASCVD risk (Table 2 in [20]). We use the pre-post within group analysis because the estimated between-group change for ASCVD risk (Table 3 in [20]) has a wider confidence interval possibly due to a relatively small sample size, less suitable controls, or other factors. For each CVD event, we calculated the associated QALYs and healthcare costs based on previously published estimates [26, 27]. The QALY losses from CVD events were calculated relative to the expected QALYs in a population of older obese women.

We conducted probabilistic sensitivity analysis to characterize parameter uncertainty in the CEAs. The analysis treats each parameter as a random variable with an assumed mean, range, and probability distribution. We conducted Monte Carlo simulations with a sample of 1000 observations, each observation representing a hypothetical trial. The simulation results are 1000 observations of incremental costs, effects, and ICERs. We graphically present the results on cost-effectiveness planes.

For the QALY CEA, we use cost-effectiveness acceptability curves to present the probability that the SHHC intervention is acceptable for a range of willingness-to-pay thresholds. The range of willingness-to-pay thresholds for QALYs includes consensus estimates of societal willingness-to-pay per QALY saved Analyses were conducted using Stata 15 (StataCorp. 2017. Stata Statistical Software: Release 15. College Station, TX: StataCorp LLC).

\section{Results}

\section{Program cost analysis}

The total costs of the direct resources used in the administration and implementation of the SHHC intervention were $\$ 78,229$ (Table 1 , all costs are in 2016 US dollars).
Table 1 Program resource costs from the payer perspective, \$

\begin{tabular}{lll}
\hline & SHHC & CON \\
\hline Cost category & & \\
Labor & 35,238 & 8563 \\
Space & 2291 & 286 \\
Food & 7182 & 995 \\
Equipment & 27,781 & 0 \\
Printing & 1336 & 195 \\
Other & 4400 & 0 \\
Total cost & 78,229 & 10,040 \\
Number of participants & 101 & 93 \\
Total cost per person & 775 & 108 \\
\hline
\end{tabular}

The resource cost per participant was $\$ 775$. The total costs of the minimal intervention education-only CON were $\$ 10,040$ and the cost per participant was $\$ 108$. From the societal perspective that includes participants' direct and opportunity costs, the cost per SHHC participant increases to $\$ 1087$ and the cost per CON participant increases to $\$ 201$ (Table 2). The largest cost component is the opportunity cost of participants' time. The Second Panel on Cost-Effectiveness in Health and Medicine [7] recommends including the opportunity costs of time, but a previous CEA of a similar intervention was not able to take them into account $[16,29,30]$. These results in Table 2 highlight the importance of the opportunity cost when evaluating participant-time intensive interventions.

\section{Intermediate health outcomes CEAs}

The calculated ICERs from the payer perspective show that the SHHC intervention costs $\$ 360$ per $\mathrm{kg}$ of weight loss, $\$ 939$ per unit of BMI reduction, $\$ 580$ per $\mathrm{mg} / \mathrm{L}$ CRP reduction, and $\$ 995$ per unit increase in Simple 7 (Table 3). The ICERs from the societal perspective are $\$ 840$ per kg of weight loss, $\$ 2187$ per unit of BMI reduction, $\$ 1351$ per $\mathrm{mg} / \mathrm{L}$ CRP reduction, and $\$ 2318$ per unit increase in Simple 7.

The variables examined in the probabilistic sensitivity analysis are presented in Tables 4 and 5 . The probabilistic sensitivity analysis show that there is considerable uncertainty in the estimated ICERs for the intermediate health outcomes (Figs. 1, 2, 3 and 4). The width (on the $\mathrm{X}$ axis) of the scatter plots of points in Figs. 1, 2, 3 and 4 reflects uncertainty about the SHHC intervention's effectiveness

Table 2 Participants' direct and opportunity costs, \$ per person

\begin{tabular}{lll}
\hline & SHHC & CON \\
\hline Cost category & & \\
Gasoline for travel in personal auto & 30 & 17 \\
Time costs & 1058 & 183 \\
Total participant cost & 1087 & 201 \\
\hline
\end{tabular}


Table 3 Cost-effectiveness of SHHC for intermediate health outcomes

\begin{tabular}{llll}
\hline & Incremental between & \multicolumn{2}{l}{$\begin{array}{l}\text { ICER from the } \\
\text { perspective of }\end{array}$} \\
\cline { 3 - 4 } & SHHC-CON & Payer & Society \\
\hline Costs, \$ per participant & & $\sqrt{ }$ & $\sqrt{ }$ \\
Resource & 667 & & $\sqrt{ }$ \\
Participant & 887 & & \\
Total & 1553 & & \\
Outcomes, per participant & & 360 & 840 \\
Weight loss, kg & -1.85 & 939 & 2187 \\
BMI reduction & -0.71 & 580 & 1351 \\
CRP reduction, mg/L & -1.15 & 995 & 2318 \\
Simple 7 increase & 0.67 &
\end{tabular}

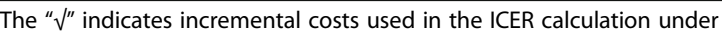
alternative perspectives

(incremental changes in the outcomes), based on the 95\% confidence intervals of the estimates from the between group multivariate analysis in the efficacy trial [20]. The height (on the $\mathrm{Y}$ axis) of the scatter plots of points reflects uncertainty about SHHC costs (incremental changes in costs from the societal perspective). To illustrate the degree of uncertainty in the resulting ICERs for weight loss, the 5 and $95 \%$ ICERs are $\$ 548$ and $\$ 1805$ per $\mathrm{kg}$ of weight loss. Note that in Table 5, the incidence rates for CHD and stroke are not the assumed CHD or stroke rates for the hypothetical population in the probabilistic sensitivity analysis. The ratio of the two incidence rates is used in the probabilistic sensitivity analysis to partition the reduction of an ASCVD event into a CHD event reduction and a stroke event reduction.

\section{QALY CEA}

From the societal perspective, which reflects all costs of the intervention to society over a 10-year time horizon, a hypothetical national SHHC is estimated to cost $\$ 238,271$ per QALY gained (Table 6). From the healthcare sector perspective, the national SHHC is estimated to cost $\$ 62,646$ per QALY.

The hypothetical national SHHC is estimated to prevent 12,768 heart events and 8512 stroke events (both non-fatal events) (Table 6). By preventing these events, SHHC is

Table 4 Parameters and variables examined in the probabilistic sensitivity analysis. Parameters with fixed values

\begin{tabular}{ll}
\hline Parameter & Value \\
\hline $\mathrm{N}$ (trials) & 1000 \\
Discount rate & 0.03 \\
Incidence rate, 55-64 Females & \\
CHD & 0.003 \\
Stroke & 0.002 \\
\hline
\end{tabular}

estimated to reduce healthcare sector costs by $\$ 857$ million, which is the healthcare cost savings that would have been spent to treat the heart and stroke events in absence of the national SHHC. However, these healthcare cost savings are not sufficient to offset the resource and participant costs (about \$4127 million) of the national intervention to a hypothetical cohort of 2.2 million women.

The probabilistic sensitivity analysis shows that there is again considerable uncertainty in the estimated societal cost per QALY gained. The results are summarized in a CEA acceptability curve, which shows the probabilities the national SHHC intervention is cost-effective at different thresholds for societal willingness-to-pay per QALY (Fig. 5). The probabilities of cost-effectiveness are measured as the fraction of estimated ICERs in the sensitivity analysis that are below each threshold.

There is not agreement on the appropriate willingnessto-pay threshold [8], but thresholds of $\$ 50,000$ and $\$ 100,000$ per QALY are commonly considered [29]. The national SHHC is very unlikely to be cost-effective at these thresholds. A recent federal guideline for regulatory impact analysis estimates willingness-to-pay per QALY based on estimates of the value of a statistical life (VSL) [30]. The range of VSL estimates imply that willingness-to-pay ranges from $\$ 230,000$ to $\$ 750,000$ per QALY; the central VSL estimate implies willingness-to-pay per QALY is $\$ 490,000$. The probability the national SHHC intervention is cost-effective is $48 \%$ using the $\$ 230,000$ threshold, $93 \%$ using the central threshold of $\$ 490,000$ and almost $97 \%$ using the $\$ 750,000$ threshold.

\section{Site-level cost-effectiveness analysis}

In addition to the overall CA and CEA of the SHHC program, which took place at eight SHHC sites (towns) and eight CON sites in Montana and New York, we estimated SHHC's costs and cost-effectiveness at the site level. The site level CA is the first step of the CA for the SHHC and CON interventions as a whole. The overall costs for SHHC and CON are simply the sum of the estimated costs in the eight SHHC sites and eight CON sites, respectively. Results of the site level CA are reported in Appendix Tables 9 and 10. The primary purpose of the site level CEA is to investigate the variation of SHHC's cost-effectiveness measured by ICERs. Unlike the overall CEA for the intermediate health outcomes (BMI, etc.), which compared SHHC with the CON, the site level CEA looked at incremental changes within group for SHHC sites (compared SHHC with status quo or the post- versus pre-intervention) for all outcomes. The site level effects of SHHC are reported in Appendix Table 11. The cost-effectiveness of SHHC in terms of ICERs is estimated from both the payer and society perspective and reported in Tables 7 and 8, respectively. 
Table 5 Parameters and variables examined in the probabilistic sensitivity analysis. Parameters with varied values

\begin{tabular}{|c|c|c|c|c|c|c|}
\hline \multirow{2}{*}{$\frac{\text { Parameter }}{\text { Outcomes }}$} & \multirow[t]{2}{*}{ Distribution } & \multirow[t]{2}{*}{ Mean } & \multicolumn{3}{|l|}{ Range } & \\
\hline & & & & & & \\
\hline BMI reduction & Normal & 0.71 & 0.08 & - & 1.35 & ( $95 \% \mathrm{Cl}$ of mean) \\
\hline Weight loss & Normal & 1.85 & 0.16 & - & 3.55 & ( $95 \% \mathrm{Cl}$ of mean) \\
\hline $\mathrm{CRP}$ reduction, $\mathrm{mg} / \mathrm{L}$ & Normal & 1.15 & 0.15 & - & 2.16 & ( $95 \% \mathrm{Cl}$ of mean) \\
\hline Simple 7 increase & Normal & 0.67 & 0.14 & - & 1.21 & ( $95 \% \mathrm{Cl}$ of mean) \\
\hline Annual ASCVD risk reduction & Log normal & 0.00096 & 0.00043 & - & 0.00149 & ( $95 \% \mathrm{Cl}$ of mean) \\
\hline \multicolumn{7}{|l|}{ Costs, per person } \\
\hline Resources of SHHC & $\curlyvee$, gamma & 775 & 581 & - & 968 & ( $\pm 25 \%$ of mean) \\
\hline Resources of SHHC-CON & $\curlyvee$, gamma & 667 & 500 & - & 833 & ( $\pm 25 \%$ of mean) \\
\hline Participant of SHHC & $Y$, gamma & 1087 & 815 & - & 1359 & ( $\pm 25 \%$ of mean) \\
\hline Participant of SHHC-CON & $\curlyvee$, gamma & 887 & 665 & - & 1108 & ( $\pm 25 \%$ of mean) \\
\hline \multicolumn{7}{|l|}{ Medical cost, $2013 \$$} \\
\hline CHD (nonfatal mi) & $\curlyvee$, gamma & 62,200 & 52,870 & - & 71,530 & $( \pm 15 \%$ of mean) \\
\hline Stroke (nonfatal) & $Y$, gamma & 20,509 & 17,433 & - & 23,585 & $( \pm 15 \%$ of mean) \\
\hline Follow-up visit, 2013 \$ & $\curlyvee$, gamma & 75 & 53 & - & 98 & $( \pm 30 \%$ of mean) \\
\hline \multicolumn{7}{|l|}{ QALYS } \\
\hline Women age 55-64, BMI 30-40 & $\beta$, beta & 0.798 & 0.678 & - & 0.918 & $( \pm 15 \%$ of mean) \\
\hline $\mathrm{CHD}$ & $\beta$, beta & 0.697 & 0.592 & - & 0.802 & $( \pm 15 \%$ of mean) \\
\hline Stroke & $\beta$, beta & 0.590 & 0.502 & - & 0.679 & $( \pm 15 \%$ of mean) \\
\hline
\end{tabular}

The incidence rates are not the assumed CHD or stroke rates for the hypothetical population in the probabilistic sensitivity analysis. The ratio of the two incidence rates is used in the probabilistic sensitivity analysis to partition the reduction of an ASCVD event into a CHD event reduction and a stroke event reduction

As in the sensitivity analysis results, the site level analysis results also show considerable variation in SHHC's costs, effects, and cost-effectiveness across sites. For example, the total resource cost per participant across SHHC sites ranges from $\$ 575$ (Site 12) up to $\$ 1106$ (Site 8) (Appendix Table 10). The change of health outcome may be in the opposite of the expected direction for all health outcomes except the Simple 7. For example, SHHC participants from Site 6 on average gained weight (instead of lost) by $1.13 \mathrm{~kg}$ after the intervention and SHHC participants from Site 16 on average had a very small increase of ASCVD risk over the next 10 years by $0.07 \%$, which results in a large ICER

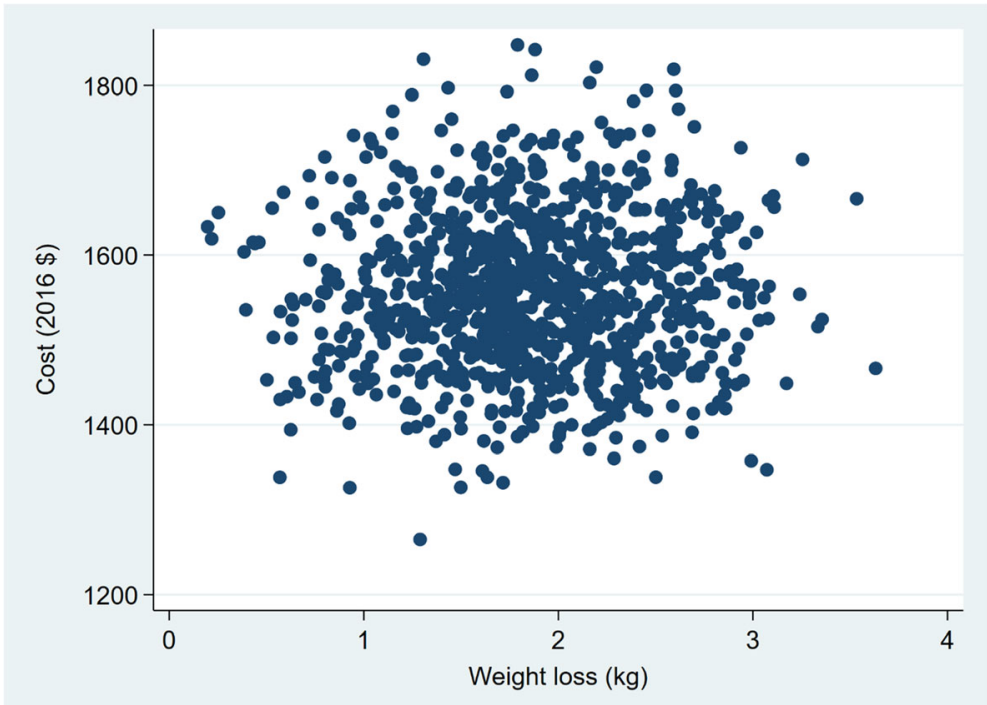

Fig. 1 Sensitivity analysis of SHHC's cost and effectiveness on weight loss 


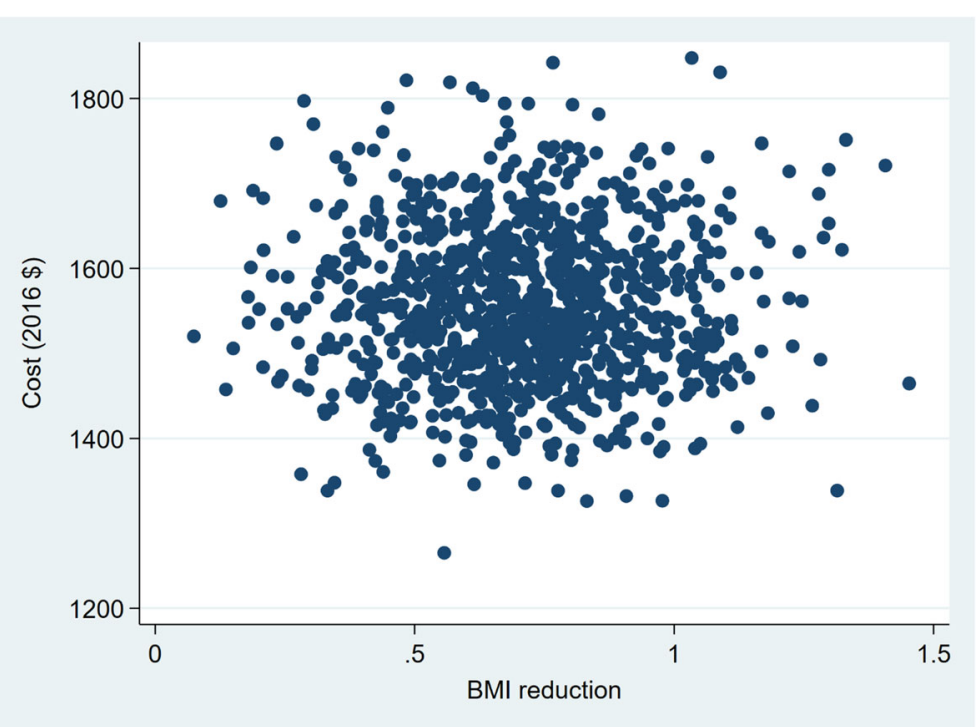

Fig. 2 Sensitivity analysis of SHHC's cost and effectiveness on BMI reduction

(3.67 million from societal perspective) for per QALY lost (Appendix Table 11). While from the societal perspective ICERs for QALY saved are relatively high in most sites, from the healthcare perspective, they are negative, indicating cost savings in Site 11 and Site 14 (Tables 7 and 8). In these two sites the SHHC resource cost is below the medical care cost that would be saved or prevented over the next 10 years per QALY saved.

\section{Discussion}

In the CA, the resource cost per SHHC participant (\$775) was somewhat higher than the costs of a few other previously studied weight loss and nutrition interventions. The average cost of providing WISEWOMAN services was $\$ 270$ per participant [31]. Because data was compiled from unique WISEWOMAN programs in multiple states, it is not possible to directly compare the program to SHHC (e.g. number of classes). An economic evaluation of the Lifestyle Education for Activity and Nutrition (LEAN) Study reported average cost per participant ranging from $\$ 54$ (multi-sensor armband only) to $\$ 365$ (multi-sensor armband plus 14 group weight loss education classes) [32]. The only previous CEA of a community-based healthy lifestyle program in

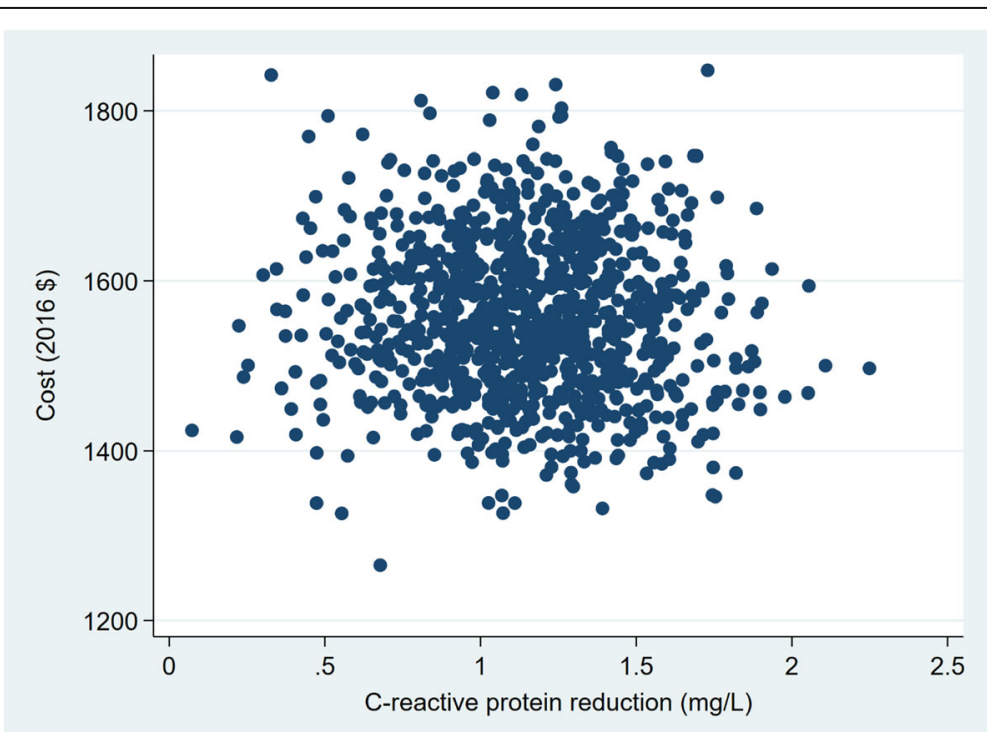

Fig. 3 Sensitivity analysis of SHHC's cost and effectiveness on CRP reduction 




Fig. 4 Sensitivity analysis of SHHC's cost and effectiveness on Simple 7 increase

the United States with midlife and older rural women, having over 50 participants was a 12-month extended care lifestyle maintenance program after an initial six-month weight loss program [16]. Participants attended twice-monthly (24 total) face-to-face group sessions or twice-monthly (24 total) individual telephone sessions or received 24 newsletters [16]. Program costs were $\$ 420$, $\$ 268$, and $\$ 226$ per participant for the face-to-face, telephone, and control programs, respectively [16]. SHHC cost $\$ 775$ per participant for the 48 sessions and the 6 -session control program cost $\$ 108$ per participant. Conversely, $\mathrm{SHHC}$ is comparable in cost per participant to an economic evaluation of the Expanded Food and Nutrition Education Program, which reported average direct (resource) costs per graduate as $\$ 715$ [33]. In this program, participants attended six or more nutrition education classes, compared to 48 classes for SHHC participants.
Adding the opportunity cost of participants' time substantially increases the costs from the societal perspective. Program administrators considering adoption of SHHC do not necessarily consider these costs because they do not have a direct impact on program budgets. However, the need to consider these costs imposed on participants is well-established in the theoretical foundations of CEA. Moreover, time costs might be an important barrier to widespread participation in an intensive intervention like SHHC.

Regarding ASCVD risk reduction, the cost-effectiveness of SHHC may be compared with the WISEWOMAN program [31, 34]. A cost-effectiveness study of the WISEWOMAN found that the program cost $\$ 4400$ (about $\$ 5300$ in 2016 dollars) per life-year gained. Calculated in a similar way, SHHC would cost about $\$ 8600$ per life-year gained. The higher cost per life-year gained in SHHC than

Table 6 Costs and effectiveness over the next 10 years of SHHC for a national sample

\begin{tabular}{|c|c|c|c|c|c|}
\hline & \multirow[t]{2}{*}{$\mathrm{SHHC}$} & \multirow[t]{2}{*}{ Status Quo } & \multirow{2}{*}{$\begin{array}{l}\text { Incremental of } \\
\text { SHHC-Status Quo }\end{array}$} & \multicolumn{2}{|c|}{ ICERs from perspective of } \\
\hline & & & & Healthcare & Society \\
\hline \multicolumn{6}{|l|}{ Costs, \$ } \\
\hline Resource & $1,716,954,549$ & 0 & $1,716,954,549$ & $\sqrt{ }$ & $\sqrt{ }$ \\
\hline Healthcare & $3,608,071,971$ & $4,465,435,607$ & $-857,363,637$ & $\sqrt{ }$ & $\sqrt{ }$ \\
\hline Participant & $2,409,841,516$ & 0 & $2,409,841,516$ & & $\sqrt{ }$ \\
\hline Total & $7,734,868,036$ & $4,465,435,607$ & $3,269,432,429$ & & \\
\hline \multicolumn{6}{|l|}{ Health effects } \\
\hline Heart events & 53,734 & 66,502 & $-12,768$ & & \\
\hline Stroke events & 35,823 & 44,335 & -8512 & & \\
\hline QALYs saved & $15,031,833$ & $15,018,112$ & 13,721 & 62,646 & 238,271 \\
\hline
\end{tabular}

" $\sqrt{ }$ " indicates incremental costs used in ICER calculation under alternative perspectives. The heart events and stroke events are non-fatal events 


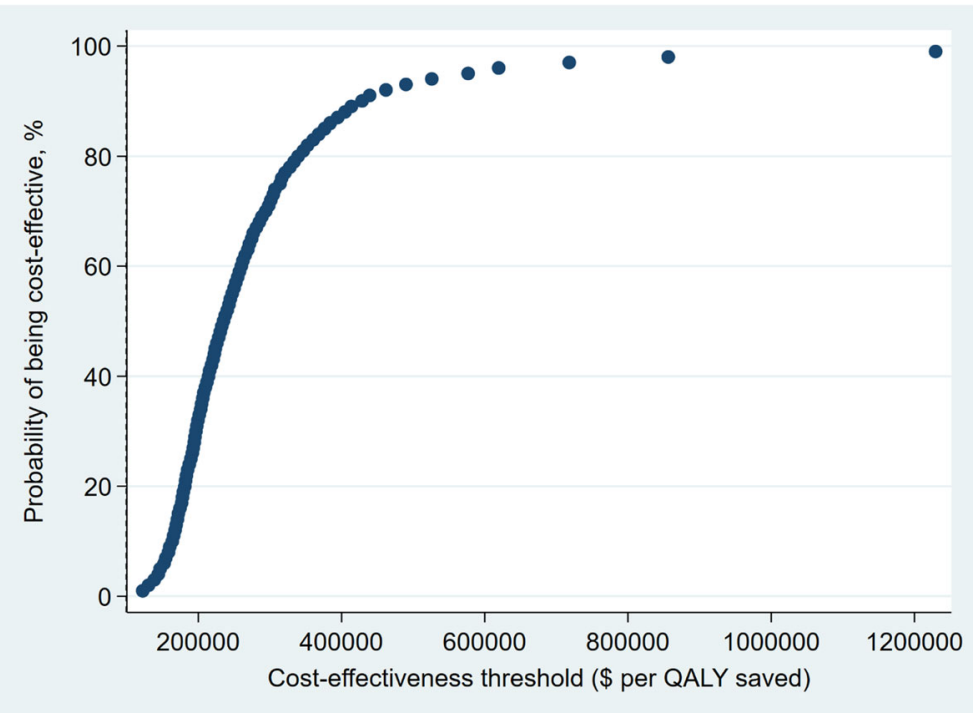

Fig. 5 Cost-effectiveness acceptability curve based on a sensitivity analysis of SHHC's cost and ASCVD risk reduction

WISEWOMAN may be related to two factors. One is that the SHHC intervention is more intensive and therefore, more costly. The other factor is the difference in sample characteristics. Compared with the WISEWOMAN sample, SHHC participants are older (average age 59 versus 52) and are already almost a completely non-smoking group (5\% versus $23 \%$ smoking). The sample difference suggests that it may be more difficult for the SHHC sample to achieve ASCVD risk improvement quickly than the WISEWOMAN sample.

By the measure of cost per QALY (or disability -adjusted life year (DALY)) saved, SHHC is less cost-effective compared with other lifestyle interventions that also help older people to lose weight $[12,13]$. Seven such studies included in two recent review studies report cost-effectiveness

Table 7 By site - Cost-effectiveness of SHHC. ICERs (vs Status Quo) from the payer or healthcare sector perspective

\begin{tabular}{llllll}
\hline Site ID & $\begin{array}{l}\text { Weight } \\
\text { loss }\end{array}$ & $\begin{array}{l}\text { BMl } \\
\text { reduction }\end{array}$ & $\begin{array}{l}\text { CRP } \\
\text { reduction }\end{array}$ & $\begin{array}{l}\text { Simple } 7 \\
\text { increase }\end{array}$ & $\begin{array}{l}\text { ASCVD QALY } \\
\text { saved }\end{array}$ \\
\hline 3 & 284 & 759 & ${ }^{*} 93,477$ & 3495 & 136,276 \\
5 & 156 & 394 & 442 & 675 & 455,779 \\
6 & $* 830$ & $* 2502$ & 482 & 1341 & 58,924 \\
8 & 343 & 954 & 921 & 921 & 186,004 \\
11 & 157 & 625 & 341 & 1811 & $-16,991$ \\
12 & 793 & 1920 & 420 & 370 & 396,841 \\
14 & 321 & 829 & 471 & 439 & -9480 \\
16 & 290 & 741 & 572 & 859 & $* 1,386,823$ \\
\hline
\end{tabular}

ICERs for QALY saved are estimated from the healthcare perspective and ICERs for other outcomes are from the payer perspective. The negative ICERs represent cost savings. The " ${ }^{* \prime \prime}$ implies that the effect is in the unexpected direction. For example, an ICER for weight loss with "*" implies that on average participants gained instead of lost body weight after the SHHC ratios ranging from about 3700US\$ (4-year time frame) per QALY to 92,100-99,200US\$ (12-month time frame) per DALY from the healthcare sector perspective [35-39]. One study reported about 13,700-15,300US\$ (6-month time frame) per life-year gained from the society perspective [40]; and another study reported about 51,700US\$ (12-month time frame) per QALY with an unclear study perspective [41]. Besides SHHC's high intensiveness and disadvantaged target population, its high estimated cost per QALY may be related to the parameters and approach used in the estimation. Other studies often use a longer time frame. We currently estimate ASCVD risk improvement for the next 10 years. If we assume the same effect on ASCVD risk improvement would continue for another 10 years (from continued lower weight, etc.), the SHHC's

Table 8 By site - Cost-effectiveness of SHHC. ICERs (vs Status Quo) from the societal perspective

\begin{tabular}{llllll}
\hline Site ID & $\begin{array}{l}\text { Weight } \\
\text { loss }\end{array}$ & $\begin{array}{l}\text { BMI } \\
\text { reduction }\end{array}$ & $\begin{array}{l}\text { CRP } \\
\text { reduction }\end{array}$ & $\begin{array}{l}\text { Simple 7 } \\
\text { increase }\end{array}$ & $\begin{array}{l}\text { ASCVD QALY } \\
\text { saved }\end{array}$ \\
\hline 3 & 683 & 1823 & $* 224,455$ & 8392 & 414,770 \\
5 & 408 & 1028 & 1153 & 1762 & $1,290,021$ \\
6 & ${ }^{*} 1692$ & $* 5099$ & 983 & 2732 & 184,905 \\
8 & 681 & 1892 & 1827 & 1827 & 430,353 \\
11 & 436 & 1729 & 945 & 5012 & 63,429 \\
12 & 2291 & 5547 & 1214 & 1068 & $1,264,574$ \\
14 & 719 & 1854 & 1055 & 983 & 56,118 \\
16 & 790 & 2022 & 1560 & 2342 & $* 3,674,482$ \\
\hline
\end{tabular}

The "*" implies that the effect is in the unexpected direction. For example, an ICER for weight loss with "*" implies that on average participants gained instead of lost body weight after the SHHC 
cost per QALY would be about $\$ 68,700$ from the societal perspective and $\$ 5300$ from the health sector perspective.

It is unknown whether CVD prevention programs similar to SHHC cost more to implement in rural versus urban areas. Urban communities are more likely to have public recreational facilities and programs [42] and may have more opportunities to form cooperative agreements and partnerships to maximize the use of facilities and staff time (e.g. with fitness centers or community organizations), reducing the overall cost of a CVD prevention program similar to SHHC.

A national SHHC intervention is likely to be cost-effective at willingness-to-pay thresholds based on guidelines for federal regulatory impact analysis. However, it is not likely to be cost-effective at commonly used lower threshold values. The cost-effectiveness of SHHC reflects a common tradeoff in the economics of prevention. Each CVD event prevented generates substantial healthcare cost savings. However, the intervention must be delivered to a large number of participants per CVD event prevented, which can be particularly difficult in medically underserved rural areas.

\section{Strengths}

This study is the first to evaluate the economic effectiveness of a multilevel community-based CVD prevention program for midlife and older women in rural, medically underserved areas via comprehensive CA and CEAs. Previous community-based lifestyle behavior change interventions in rural areas lacked comparison groups; had small sample sizes; and/or included younger adults, both men and women; or included telephone-based programs. Additionally, previous studies did not include participant time costs.

\section{Limitations}

SHHC was a multilevel intervention, which required additional staff and participant time, and included social and community components that were not measured. Primary outcomes for SHHC were measured at six months; future CEAs should collect data that would enable measurement of the longer-term impact on individuals, as well as on social and community components targeted by SHHC.

One limitation of our study is not having converted all of the intervention benefits into QALYs, which can be used to compare with a wider range of interventions. The estimated QALYs saved in our analysis come from the reduced ASCVD risk by the SHHC program. Other benefits of the SHHC program may also save QALYs. For example, the SHHC program reduces BMI, which may prevent diabetes and save QALYs.

Our assumption that with the initial intervention cost of $\$ 775$, the intervention's benefit of ASCVD risk reduction would last for the next 10 years is not entirely reasonable due to the likelihood of weight regain or changes in other relevant factors. Additional costs may be incurred to maintain participants' healthier status and ASCVD benefit but they may also increase the total intervention cost. Not taking into account the possible future costs is a limitation of this study.

We attribute the change in ASCVD risk scores pre and post the SHHC program as the causal effect of the intervention. This approach of using the prediction model for ASCVD risk score in a causal sense has limitations. This is a general problem with chronic disease prevention: it is costly and takes decades to run randomized controlled trials with hard ASCVD events as outcomes, so it is common to use epidemiologic modelling to translate the changes in outcomes measured in the randomized controlled trials into the changes in health outcomes and QALYs.

Another limitation of our study is not using the lifetime frame for the estimation of the benefits and costs of the reduced ASCVD risk by the SHHC program. We use the 10-year frame because the ASCVD risk measure is for a 10 -year period. The limited time horizon may result in under- or over-estimated cost-effectiveness of the SHHC on ASCVD risk.

The last limitation of our study is that we do not have cost information for the ASCVD events for our study population. However, our estimates of ICERs for QALY saved are not very sensitive to the medical costs. For example, the ICERs for QALYs saved decrease slightly (more from the health sector perspective than from the societal perspective) if we increase the assumed stroke event cost from $\$ 20,509$ to $\$ 50,000$.

\section{Recommendations}

To improve the cost-effectiveness of SHHC, the program could be adapted to achieve greater impacts on weight, Simple 7, and ASCVD as well as to further impact additional individuals (through individual, social, or community components). The intervention could also reduce staff from one educator and one program assistant down to just one educator. Participant time or other costs, such as space rental, could also be reduced. To impact more individuals, participants could attend classes with a friend or family member.

\section{Conclusions}

The results of the economic evaluations of the SHHC intervention are informative for U.S. health policy. Policy makers should give a higher priority to implement other more cost-effective interventions, but the SHHC intervention still represents a reasonable return on investment. There might also be opportunities to better target the intervention to improve cost-effectiveness and it may be that programs similar to SHHC are more expensive to operate in rural areas. 


\section{Appendix}

Table 9 By site - Cost by categories, \$

\begin{tabular}{|c|c|c|c|c|c|c|c|c|c|c|}
\hline \multirow[t]{2}{*}{ Group } & \multirow{2}{*}{$\begin{array}{l}\text { Site } \\
\text { ID }\end{array}$} & \multirow[t]{2}{*}{ Enrollees } & \multicolumn{6}{|c|}{ Resource cost } & \multicolumn{2}{|c|}{ Participant cost } \\
\hline & & & Food & Labor & Space & Other & Equipment & Printing & Gas & Time \\
\hline CON & 1 & 14 & 199 & 424 & 36 & 0 & 0 & 24 & 641 & 1496 \\
\hline CON & 2 & 13 & 156 & 768 & 36 & 0 & 0 & 24 & 145 & 2096 \\
\hline CON & 7 & 9 & 51 & 528 & 36 & 0 & 0 & 24 & 49 & 3004 \\
\hline CON & 9 & 12 & 98 & 1772 & 36 & 0 & 0 & 24 & 82 & 1575 \\
\hline CON & 10 & 11 & 148 & 1860 & 36 & 0 & 0 & 24 & 191 & 2016 \\
\hline $\mathrm{CON}$ & 13 & 10 & 93 & 1070 & 36 & 0 & 0 & 24 & 173 & 1833 \\
\hline $\mathrm{CON}$ & 15 & 12 & 125 & 1070 & 36 & 0 & 0 & 24 & 208 & 2199 \\
\hline $\mathrm{CON}$ & 17 & 12 & 125 & 1070 & 36 & 0 & 0 & 24 & 208 & 2199 \\
\hline $\mathrm{SHHC}$ & 3 & 13 & 992 & 4405 & 50 & 1000 & 3473 & 167 & 385 & 13,748 \\
\hline $\mathrm{SHHC}$ & 5 & 13 & 1016 & 3424 & 150 & 550 & 3473 & 167 & 385 & 13,748 \\
\hline $\mathrm{SHHC}$ & 6 & 12 & 959 & 5512 & 1150 & 0 & 3473 & 167 & 165 & 11,520 \\
\hline $\mathrm{SHHC}$ & 8 & 12 & 1212 & 6440 & 475 & 1500 & 3473 & 167 & 355 & 12,690 \\
\hline $\mathrm{SHHC}$ & 11 & 13 & 851 & 4288 & 50 & 0 & 3473 & 167 & 592 & 15,015 \\
\hline $\mathrm{SHHC}$ & 12 & 12 & 306 & 2360 & 100 & 500 & 3473 & 167 & 355 & 12,690 \\
\hline $\mathrm{SHHC}$ & 14 & 11 & 781 & 4405 & 286 & 550 & 3473 & 167 & 326 & 11,633 \\
\hline $\mathrm{SHHC}$ & 16 & 15 & 1066 & 4405 & 30 & 300 & 3473 & 167 & 444 & 15,863 \\
\hline
\end{tabular}

Table 10 By site - Total costs per participant, \$

\begin{tabular}{llll}
\hline Group & Site ID & Resources & Resources and participant \\
\hline CON & 1 & 49 & 201 \\
CON & 2 & 76 & 248 \\
CON & 7 & 71 & 410 \\
CON & 9 & 161 & 299 \\
CON & 10 & 188 & 389 \\
CON & 13 & 122 & 323 \\
CON & 15 & 105 & 305 \\
CON & 17 & 105 & 305 \\
SHHC & 3 & 776 & 1863 \\
SHHC & 5 & 675 & 1762 \\
SHHC & 6 & 938 & 1912 \\
SHHC & 8 & 1106 & 2193 \\
SHHC & 11 & 679 & 1880 \\
SHHC & 12 & 575 & 1663 \\
SHHC & 14 & 878 & 1965 \\
SHHC & 16 & 629 & 1716 \\
\hline
\end{tabular}


Table 11 By site - Effects of SHHC (vs Status Quo), per participant

\begin{tabular}{llllllll}
\hline Site ID & Weight, kg & BMI & CRP & Simple 7 & ASCVD risk, & QALYs & Medical cost, \\
\hline 3 & -2.73 & -1.02 & 0.01 & 0.22 & -0.61 & 0.0039 & -244 \\
5 & -4.32 & -1.71 & -1.53 & 1 & -0.2 & 0.0013 & -81 \\
6 & 1.13 & 0.38 & -1.95 & 0.7 & -1.2 & 0.0077 & -483 \\
8 & -3.22 & -1.16 & -1.2 & 1.2 & -0.69 & 0.0044 & -278 \\
11 & -4.32 & -1.09 & -1.99 & 0.38 & -2.32 & 0.0149 & -933 \\
12 & -0.73 & -0.3 & -1.37 & 1.56 & -0.19 & 0.0013 & -78 \\
14 & -2.73 & -1.06 & -1.86 & 2 & -2.57 & 0.0166 & -1035 \\
16 & -2.17 & -0.85 & -1.1 & 0.73 & 0.07 & -0.0005 & 30 \\
\hline
\end{tabular}

Notes: Changes for ASCVD risk, QALYs saved, and Medical cost are for the next 10 years

\section{Abbreviations}

ASCVD: Atherosclerotic cardiovascular disease; CA: Cost analysis; CEA: Costeffectiveness analysis; CHD: Coronary heart disease; CON: Minimal, educationonly control program; CRP: C-reactive protein; CVD: Cardiovascular disease; DALY: Disability-adjusted life year; ICER: Incremental cost-effectiveness ratio; LEAN: Lifestyle Education for Activity and Nutrition; QALY: Quality-adjusted life year; SHHC: Strong Hearts, Healthy Communities; VSL: Value of a statistical life

\section{Acknowledgments}

We would like to acknowledge our funding from the National Heart, Lung and Blood Institute (R01 HL120702). We would also like to the contributions and efforts of our National Advisory Board members, as well as the contributions and efforts of our program leaders on the study and the study participants in both states, without whom this research would not be possible.

\section{Funding}

This study was supported by grant R01 HL120702 from the National Institutes of Health and the National Heart, Lung and Blood Institute (NHLBI). The funders had no role in the design and conduct of the study; collection, management, analysis, or interpretation of the data; preparation, review, or approval of the manuscript; or decision to submit the manuscript for publication.

\section{Availability of data and materials}

The datasets used for the current study are available from the corresponding author on reasonable request.

\section{Authors' contributions}

HW and DK analyzed and interpreted cost and cost-effectiveness data and were major contributors in writing the manuscript. RAS conceived of and is overseeing all aspects of the study including writing of this manuscript. MLG lead data collection for this manuscript; she also drafted and revised portions of the manuscript. SCF, MEN, and LCP participated in study design and reviewed and approved the final manuscript. DS participated in study design, supported study implementation, and has reviewed and approved the final manuscript. All authors read and approved the final manuscript.

\section{Ethics approval and consent to participate}

The study was approved by the Cornell University and Bassett Healthcare Institutional Review Boards. Participants provided signed informed consent.

\section{Consent for publication}

Not applicable.

\section{Competing interests}

The authors declare that they have no competing interests.

\section{Publisher's Note}

Springer Nature remains neutral with regard to jurisdictional claims in published maps and institutional affiliations.

\section{Author details}

'Cornell University, 292 Martha Van Rensselaer Hall, Ithaca, NY 14853, USA.

${ }^{2}$ Cornell University, 2310 Martha Van Rensselaer Hall, Ithaca, NY 14853, USA

${ }^{3}$ Cornell University, 414 Savage Hall, Ithaca, NY 14853, USA. ${ }^{4}$ Montana State University, 322 Reid Hall, Bozeman, MT 59717, USA. ${ }^{5}$ Tufts University, 150 Harrison Ave, Boston, MA 02111, USA. 'Hampshire College, 893 West St,

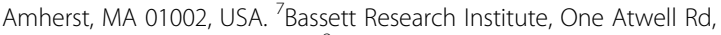
Cooperstown, NY 13326, USA. ${ }^{8}$ Cornell University, 412 Savage Hall, Ithaca, NY 14853, USA.

Received: 20 November 2018 Accepted: 22 April 2019

Published online: 16 May 2019

\section{References}

1. Benjamin EJ, Virani SS, Callaway CW, Chamberlain AM, Chang AR, Cheng S, et al. Heart disease and stroke statistics - 2018 update: a report from the American Heart Association. Circulation. 2018;137(12):E67-E492.

2. Meit M, Knudson A, Gilbert T, Tzy-Chyi Yu A, Tanenbaum E, Ormson E, et al. A 2014 update of the rural-urban Chartbook: Rural Health Reform Policy Research Center; 2014.

3. O'Connor A, Wellenius G. Rural-urban disparities in the prevalence of diabetes and coronary heart disease. Public Health. 2012;126(10):813-20.

4. Thornton L, Crawford D, Cleland V, Timperio A, Abbott G, Ball K. Do food and physical activity environments vary between disadvantaged urban and rural areas? Findings from the READI study. Health Promot J Austr. 2012; 23(2):153-6.

5. Michimi A, Wimberly MC. Natural environments, obesity, and physical activity in nonmetropolitan areas of the United States. J Rural Health. 2012; 28(4):398-407.

6. Olsen J. An integrative review of literature on the determinants of physical activity among rural women. Public Health Nurs. 2013;30(4):288-311.

7. Sanders GD, Neumann PJ, Basu A, Brock DW, Feeny D, Krahn M, et al. Recommendations for conduct, methodological practices, and reporting of cost-effectiveness analyses second panel on cost-effectiveness in health and medicine. JAMA. 2016;316(10):1093-103.

8. Neumann P, Sanders G, Russell L, Siegel J, Ganiats T. Cost-effectiveness in health and medicine. 2nd ed. New York: Oxford University Press; 2017.

9. Maciosek MV, Coffield AB, Flottemesch TJ, Edwards NM, Solberg LI. Greater use of preventive services in US health care could save lives at little or no cost. Health Aff. 2010;29(9):1656-60.

10. Russell LB. Preventing chronic disease: an important investment, but don't count on cost savings. Health Aff. 2009:28(1):42-5.

11. Lehnert T, Sonntag D, Konnopka A, Riedel-Heller S, Konig H. The long-term cost-effectiveness of obesity prevention interventions: systematic literature review. Obes Rev. 2012;13(6):537-53.

12. Alouki K, Delisle H, Bermudez-Tamayo C, Johri M. Lifestyle Interventions to Prevent Type 2 Diabetes: A Systematic Review of Economic Evaluation Studies. Journal of Diabetes Research. 2016;2016:14. Article ID 2159890. https://doi.org/10.1155/2016/2159890.

13. Saha S, Gerdtham U, Johansson P. Economic evaluation of lifestyle interventions for preventing diabetes and cardiovascular diseases. Int J Environ Res Public Health. 2010;7(8):3150-95.

14. Smith KJ, Hsu HE, Roberts MS, Kramer MK, Orchard TJ, Piatt GA, Seidel MC Zgibor JC, Bryce CL. Cost-Effectiveness Analysis of Efforts to Reduce Risk of 
Type 2 Diabetes and Cardiovascular Disease in Southwestern Pennsylvania 2005-2007. Prev Chronic Dis. 2010;7(5):A109. https://www.ncbi.nlm.nih.gov/ pubmed/20712936.

15. Perri MG, Limacher MC, von Castel-Roberts K, Daniels MJ, Durning PE, Janicke DM, et al. Comparative effectiveness of three doses of weightloss counseling: two-year findings from the rural LITE trial. Obesity. 2014;22(11):2293-300

16. Radcliff $T$, Bobroff $L$, Lutes $L$, Durning $P$, Daniels $M$, Limacher $M$, et al. Comparing costs of telephone vs face-to-face extended-care programs for the management of obesity in rural settings. J Acad Nutr Diet. 2012;112(9):1363-73.

17. Befort C, Donnelly J, Sullivan D, Ellerbeck E, Perri M. Group versus individual phone-based obesity treatment for rural women. Eat Behav. 2010;11(1):11-7.

18. Schrock LE. Review of cost efficiency and efficacy of delivering a diabetes education program in a southwest rural healthcare facility. Diabetes Educ. 1998;24(4):485-92.

19. Seguin RA, Eldridge G, Graham ML, Folta SC, Nelson ME, Strogatz D. Strong Hearts, Healthy Communities: A Rural Community-Based Cardiovascular Disease Prevention Program. BMC Public Health. 2016;16:86. https://doi.org/ 10.1186/s12889-016-2751-4

20. Seguin RA, Paul L, Folta SC, Nelson ME, Strogatz D, Graham ML, et al. Strong hearts, healthy communities: a community-based randomized trial for rural women. Obesity. 2018;26(5):845-53.

21. Seguin R, Heidkamp-Young E, Kuder J, Nelson M. Improved physical fitness among older female participants in a nationally disseminated, communitybased exercise program. Health Educ Behav. 2012;39(2):183-90.

22. Folta SC, Lichtenstein AH, Seguin RA, Goldberg JP, Kuder JF, Nelson ME. The StrongWomen-healthy hearts program: reducing cardiovascular disease risk factors in rural sedentary, overweight, and obese midlife and older women. Am J Public Health. 2009:99(7):1271-7.

23. Seguin RA, Folta SC, Sehlke M, Nelson ME, Heidkamp-Young E, Fenton M, Junot B. The StrongWomen Change Clubs: Engaging Residents to Catalyze Positive Change in Food and Physical Activity Environments. Journal of Environmental and Public Health. 2014;2014:6. Article ID 162403. https://doi. org/10.1155/2014/162403.

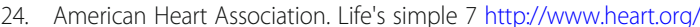
HEARTORG/Conditions/My-Life-Check_Lifes-Simple-7_UCM_471453_Article. jsp. Accessed 19 Nov 2018

25. Goff DC Jr, Lloyd-Jones DM, Bennett G, Coady S, D'Agostino RB, Gibbons R, Greenland P, Lackland DT, Levy D, O'Donnell CJ, Robinson JG, Schwartz JS, Shero ST, Smith SC Jr, Sorlie P, Stone NJ, Wilson PW, Jordan HS, Nevo L, Wnek J, Anderson JL, Halperin JL, Albert NM, Bozkurt B, Brindis RG, Curtis LH, DeMets D, Hochman JS, Kovacs RJ, Ohman EM, Pressler SJ, Sellke FW, Shen WK, Smith SC Jr, Tomaselli GF, and Guidelines American College of Cardiology/American Heart Association Task Force on Practice. 2013 Acc/ Aha Guideline on the Assessment of Cardiovascular Risk: A Report of the American College of Cardiology/American Heart Association Task Force on Practice Guidelines. Circulation. 2014;129(25 Suppl 2):S49-73. https://doi.org/ 10.1161/01.cir.0000437741.48606.98.

26. Brown $M$, Marsh $T$, Retat $L$, Fordham $R$, Suhrcke $M$, Turner $D$, et al. Managing overweight and obesity among adults: report on economic modelling and cost consequence analysis. UK: NICE; 2013.

27. O'Sullivan AK, Rubin J, Nyambose J, Kuznik A, Cohen DJ, Thompson D. Cost estimation of cardiovascular disease events in the US. Pharmacoeconomics. 2011;29(8):693-704.

28. Carlson SA, Densmore D, Fulton JE, Yore MM, Kohl HW. Differences in physical activity prevalence and trends from 3 U.S. surveillance systems: NHIS, NHANES, and BRFSS. J Phys Act Health. 2009;6:S18-27.

29. Pandya A, Sy S, Cho S, Weinstein MC, Gaziano TA. Cost-effectiveness of 10year risk thresholds for initiation of statin therapy for primary prevention of cardiovascular disease. JAMA. 2015;314(2):142-50.

30. Office of the Assistance Secretary for Planning and Evaluation, U.S Department of Health and Human Services (2016). Guidelines for Regulatory Impact Analysis. Retrieved from: https://aspe.hhs.gov/system/files/pdf/ 242926/HHS_RIAGuidance.pdf. Accessed 29 Apr 2019.

31. Finkelstein EA, Khavjou O, Will JC. Cost-effectiveness of WISEWOMAN, a program aimed at reducing heart disease risk among low-income women. J Women's Health. 2006:15(4):379-89.

32. Archer E, Groessl EJ, Sui XM, McClain AC, Wilcox S, Hand GA, et al. An economic analysis of traditional and technology-based approaches to weight loss. Am J Prev Med. 2012;43(2):176-82.
33. Dollahite J, Kenkel D, Thompson CS. An economic evaluation of the expanded food and nutrition education program. J Nutr Educ Behav. 2008; 40(3):134-43.

34. Finkelstein EA, Troped PJ, Will JC, Palombo R. Cost-effectiveness of a cardiovascular disease risk reduction program aimed at financially vulnerable women: the Massachusetts WISEWOMAN project. J Womens Health Gend Based Med. 2002;11(6):519-26.

35. Tsai AG, Wadden TA, Volger $S$, Sarwer DB, Vetter M, Kumanyika S, et al. Costeffectiveness of a primary care intervention to treat obesity. Int I Obesity. 2013;37:S31-S7

36. Forster M, Veerman $\mathrm{JL}$, Barendregt JJ, Vos T. Cost-effectiveness of diet and exercise interventions to reduce overweight and obesity. Int J Obesity. 2011; 35(8):1071-8.

37. Bertram MY, Lim SS, Barendregt JJ, Vos T. Assessing the cost-effectiveness of drug and lifestyle intervention following opportunistic screening for prediabetes in primary care. Diabetologia. 2010;53(5):875-81.

38. Cobiac $L$, Vos T, Veerman L. Cost-effectiveness of weight watchers and the lighten up to a healthy lifestyle program. Aust N Z J Public Health. 2010; 34(3):240-7.

39. Sagarra R, Costa B, Cabre JJ, Sola-Morales O, Barrio F, Grp Invest D-P-CP. Lifestyle interventions for diabetes mellitus type 2 prevention. Rev Clin Esp. 2014:214(2):59-68

40. Lindgren P, Fahlstadius P, Hellenius ML, Jonsson B, de Faire U. Costeffectiveness of primary prevention of coronary heart disease through risk factor intervention in 60-year-old men from the county of Stockholm - a stochastic model of exercise and dietary advice. Prev Med. 2003:36(4):403-9.

41. McConnon A, Kirk SF, Cockroft JE, Harvey EL, Greenwood DC, Thomas JD, Ransley JK, Bojke L. The Internet for Weight Control in an Obese Sample: Results of a Randomised Controlled Trial. BMC Health Serv Res. 2007;7:206. https://doi.org/10.1186/1472-6963-7-206.

42. Quinn C, Slater S, Barker D, Chaloupka F. Availability of local public recreational facilities and programs for physical activity. A BTG research brief. Chicago: Bridging the Gap Program, Health Policy Center, Institute for Health Research and Policy, University of Illinois at Chicago; 2015.

Ready to submit your research? Choose BMC and benefit from

- fast, convenient online submission

- thorough peer review by experienced researchers in your field

- rapid publication on acceptance

- support for research data, including large and complex data types

- gold Open Access which fosters wider collaboration and increased citations

- maximum visibility for your research: over $100 \mathrm{M}$ website views per year

At $\mathrm{BMC}$, research is always in progress.

Learn more biomedcentral.com/submission 